\title{
Propagation of Long Fatigue Cracks under Remote Modes II and III in Ferritic-Pearlitic Steel
}

\author{
T. Vojtek ${ }^{a, b, *}$, J. Pokluda ${ }^{a, b}$, A. Hohenwarter ${ }^{c}$ And R. Pippan ${ }^{d}$ \\ ${ }^{a}$ Faculty of Mechanical Engineering, Brno University of Technology, Technická 2, CZ-61669 Brno, Czech Republic \\ ${ }^{b}$ Central European Institute of Technology (CEITEC), Brno University of Technology, \\ Technická 10, CZ-61600 Brno, Czech Republic \\ ${ }^{c}$ Department of Materials Physics, Montanuniversität Leoben, Jahnstr. 12, A-8700 Leoben, Austria \\ ${ }^{d}$ Erich Schmid Institute of Materials Science, Austrian Academy of Sciences, Jahnstr. 12, A-8700 Leoben, Austria
}

\begin{abstract}
The aim of this research was to determine the 3D crack path and the related growth micromechanism in specimens made of the ferritic-pearlitic steel loaded in modes II and III. Crack closure effects were minimized at the beginning of the experiments by a special technique of generation of precracks in order to measure effective values of fatigue thresholds. Dependences of the crack growth rate on the stress intensity factor range were plotted for the near-threshold region. The effective mode III threshold $\Delta K_{\mathrm{IIIeff}, \text { th }}=4.4 \mathrm{MPa} \mathrm{m}^{1 / 2}$ was found to be higher than that for mode II $\left(\Delta K_{\text {IIeff,th }}=2.9 \mathrm{MPa} \mathrm{m}^{1 / 2}\right.$, the theory predicts $\left.3.1 \mathrm{MPa} \mathrm{m}^{1 / 2}\right)$ which is in agreement with the results for other metallic materials and available theoretical models. Fractographical observations in three dimensions revealed that the remote mode II cracks deflected towards the opening mode I by the mean angle $\approx 50^{\circ}$, which is significantly different from the low-angle deflections (coplanar growth) previously observed in the ARMCO iron. The simple linear elastic fracture mechanics based criterion for growth of a mode I branch at the crack front loaded in mode II was fulfilled.
\end{abstract}

DOI: $10.12693 /$ APhysPolA.128.611

PACS: $62.20 . \mathrm{Mk}, 81.40 . \mathrm{Np}$

\section{Introduction}

The threshold of the stress intensity factor range (SIFR) is an important mechanical property of a material that, in the small-scale yielding case, determines the conditions from non-propagation to propagation of a cyclically loaded crack. It is useful to distinguish the extrinsic from the intrinsic component not only under mode I loading $[1,2]$ but also under shear-modes II and III loadings. In the latter cases, the extrinsic component can play a dominant role especially in the near-threshold region. The intrinsic (effective) mechanisms are responsible for the generation of new fracture surfaces at the crack tip, i.e., the fatigue crack propagation. The extrinsic (shielding) mechanisms occur in the surrounding of the crack tip as contacts of fracture surfaces during the cyclic loading (crack closure).

There is an insufficient knowledge of basic mechanisms of the intrinsic resistance to shear-mode crack growth.

TABLE I

Effective thresholds $\Delta K_{\text {IIeff,th }}$ and $\Delta K_{\text {IIIeff,th }}$ [7].

\begin{tabular}{c|c|c}
\hline \hline \multirow{2}{*}{ Material } & $\Delta K_{\text {IIth,eff }}$ & $\Delta K_{\text {IIIth,eff }}$ \\
\cline { 2 - 3 } & \multicolumn{2}{|c}{$\left[\mathrm{MPa} \mathrm{m}^{1 / 2}\right]$} \\
\hline ARMCO iron & 1.5 & 2.6 \\
titanium & 1.7 & 2.8 \\
stainless steel X5CrNi18-10 & 2.5 & 4.2 \\
nickel & 2.9 & 4.3
\end{tabular}

* corresponding author; e-mail:

tomas.vojtek@ceitec.vutbr.cz
A few years ago, there was a complete lack of experimental data on effective thresholds $\Delta K_{\text {IIeff,th }}$ and $\Delta K_{\text {IIIeff,th }}$ and only a few data [3-5] available on crack-growth rates in the near-threshold and Paris-Erdogan region. Recently $[6,7]$, this unpleasant situation was improved by obtaining experimental data on $\Delta K_{\text {IIeff,th }}$ and $\Delta K_{\text {IIIeff,th }}$ for several single-phase materials - see Table I.

The data for $\Delta K_{\text {IIeff,th }}$ were well reproduced by the following simple formula:

$$
\Delta K_{\text {IIeff,th }}=G b^{1 / 2} / n_{\alpha},
$$

where $G$ is the shear modulus, $b$ is the magnitude of Burgers vector,

$$
n_{\alpha}=\frac{1}{2} \cos \left(\alpha_{\operatorname{IIm}} / 2\right)\left(3 \cos \alpha_{\operatorname{IIm}}-1\right),
$$

and $\alpha_{\mathrm{IIm}}$ is the mean deflection angle of the crack front from the plane of the maximum shear stress [7]. For the investigated single-phase materials, the angle $\alpha_{\text {IIm }}$ increased with decreasing number of available easy slip systems in the crystal lattice and $n_{\alpha, \mathrm{bcc}} \approx 0.9, n_{\alpha, \mathrm{hcp}} \approx 0.6$, and $n_{\alpha, \text { fcc }} \approx 0.4$ for bcc, hcp, and fcc metals, respectively.

The theoretical models $[8,9]$ predict faster crack growth rate in the mode II than that in the mode III for metallic materials and, accordingly, the ratio of effective thresholds $\Delta K_{\text {IIIeff, th }} / \Delta K_{\text {IIeff,th }}>1$. Indeed, the measured ratio $\Delta K_{\text {IIIeff, th }} / \Delta K_{\text {IIeff,th }} \approx 1.7$ was found for all of the four investigated single-phase materials (see Table I) and, simultaneously, the validity of Eq. (1) was also confirmed [7]. However, a majority of engineering materials exhibit a multi-phase microstructure and, therefore, a ferritic-pearlitic steel was experimentally studied in this work. 


\section{Experimental \\ 2.1. Material and specimens}

Modes II and III fatigue crack growth in experiments were done on the ferritic-pearlitic steel C45 (ISO C60E4, $0.45 \mathrm{wt} \% \mathrm{C})$. Three different specimens were used: (i) the compact-tension shear specimens (CTS) with the dimensions of $68 \times 42 \mathrm{~mm}^{2}$ and the thickness of $4 \mathrm{~mm}$ for pure mode II loading, (ii) the cylindrical bar with a circumferential notch with the outer diameter of $25 \mathrm{~mm}$, the inner diameter of $12 \mathrm{~mm}$ and the length of $100 \mathrm{~mm}$ loaded in torsion to generate a pure mode III loading and (iii) the same cylindrical bar installed into a special device transforming tensile forces into a pure shear loading of the circular notch which resulted in a combination of loading modes II, III and II + III. A more detailed description of the specimens can be found in [6]. Precracks were made at the notch roots of the specimens in cyclic compression to suppress the friction induced crack closure effects at the beginning of the shear-mode experiments. The specimens were annealed to minimize the effect of plastic zone created after precracking. After the experiments the specimens were fractured in mode I.

\subsection{Crack growth rates and thresholds}

The modes II and III crack lengths were measured on fracture surfaces after the experiments in the scanning electron microscope (SEM). The averaged crack growth rate data for all three types of specimens are presented in Fig. 1 in terms of $\Delta a / \Delta N$ vs. $\Delta K$ curves. Note that only the threshold values $\Delta K_{\text {IIeff,th }}$ and $\Delta K_{\text {IIIeff,th }}$ (related to $\Delta a / \Delta N=10^{-13} \mathrm{~m} /$ cycle at the $\Delta a / \Delta N$ vs. $\Delta K$ curves in Fig. 1) can be considered to be intrinsic. Other points correspond to shear-mode crack propagation during which a friction-induced crack closure is already developed. The data were fitted using the equation $\Delta a / \Delta N=A\left(\Delta K^{n}-\Delta K_{\mathrm{th}}^{n}\right)$ suitable for the nearthreshold region [10].

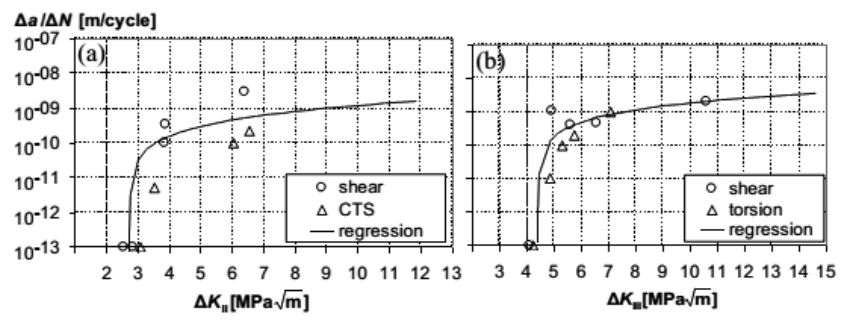

Fig. 1. Crack growth rate data for the ferritic-pearlitic steel in modes II (a) and III (b).

Despite a high scatter of the data in Fig. 1, the threshold values can be estimated as $\Delta K_{\text {IIeff,th }}=2.9 \mathrm{MPa} \mathrm{m}^{1 / 2}$ and $\Delta K_{\text {IIIeff }, \text { th }}=4.4 \mathrm{MPa} \mathrm{m}^{1 / 2}$. These values are higher than those for ferrite.

\subsection{Fractography}

Under the remote mode II loading, the shear-mode crack growth often switches to mixed mode I + II local propagation or to a pure mode I growth when deflecting (bifurcating) from the plane of a maximum shear stress. In the latter case the related deflection angle of the elementary branch is $\alpha_{\text {IIc }}=70.5^{\circ}$ (e.g. [11]). Similarly, the mode III cracks can transfer to the mixed mode I + III or to a pure mode I growth when twisting from the maximum shear plane by the angle $\beta_{\text {III }}\left(\beta_{\text {IIc }}=45^{\circ}\right)$. The deflection angles $\alpha_{\text {II }}$ and $\beta_{\text {III }}$ are schematically depicted in Fig. 2. Their values determine the amount of local mode I support to shear-mode crack propagation. For the ARMCO iron (pure ferrite), these angles were found to be very small (nearly coplanar crack growth). The angles in titanium and nickel were rather higher but still smaller than the value for pure mode I branches which were detected only in the stainless steel.

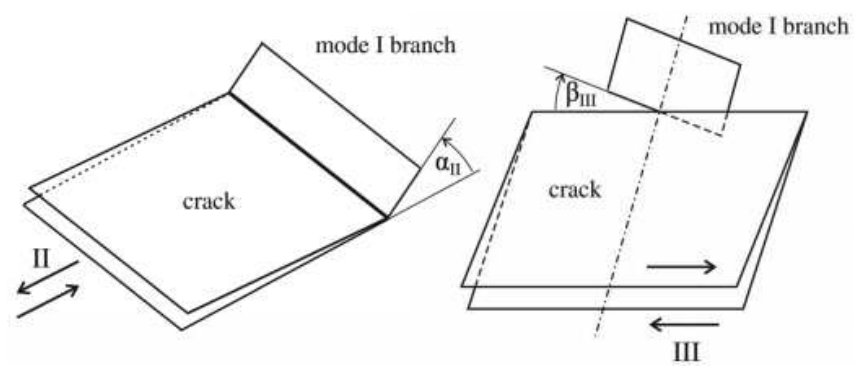

Fig. 2. Schematic depiction of a deflection of the remote mode II loaded crack by an angle $\alpha_{\text {II }}$ creating a mode I branch and of a twist of the remote mode III loaded crack by an angle $\beta_{\text {III }}$ creating a mode I branch.

TABLE II

Mean deflection and twist angles [ ${ }^{\circ}$ ] with standard deviations obtained from the height profiles measured on the fracture surfaces.

\begin{tabular}{c|c|c|c|c}
\hline \hline \multicolumn{2}{c|}{} & \multicolumn{2}{c}{ specimen } \\
\cline { 4 - 5 } \multicolumn{2}{c|}{} & shear & CTS/torsion \\
\hline \multirow{2}{*}{ II } & $\alpha_{\text {II }}$ & deflection & $48 \pm 18$ & $52 \pm 9$ \\
\cline { 2 - 5 } & $\beta_{\text {II }}$ & twist & $23 \pm 13$ & $28 \pm 9$ \\
\hline \multirow{2}{*}{ III } & $\beta_{\text {III }}$ & twist & $33 \pm 18$ & $29 \pm 17$ \\
\cline { 2 - 5 } & $\alpha_{\text {III }}$ & deflection & $23 \pm 14$ & $34 \pm 16$
\end{tabular}

For the ferritic-pearlitic steel the fracture surfaces were reconstructed in three dimensions using stereophotogrammetry in SEM. Figure 3 shows examples of the fractographs with the height coordinate $z$ visible in the color codes and in the height profiles running parallel to the applied shear along the coordinate $l$. The height profiles enabled measurements of the deflection angles $\alpha_{\mathrm{II}}$ of the mode II cracks and the twist angles $\beta_{\text {III }}$ of the mode III cracks. Their mean values are summarized in Table II. In addition, mean values of the deflection angles $\alpha_{\text {III }}$ of the mode III cracks and the twist angles $\beta_{\text {II }}$ of the mode II cracks are also presented in Table II, however these values do not have any direct relation to the physical mechanism of the crack propagation. 

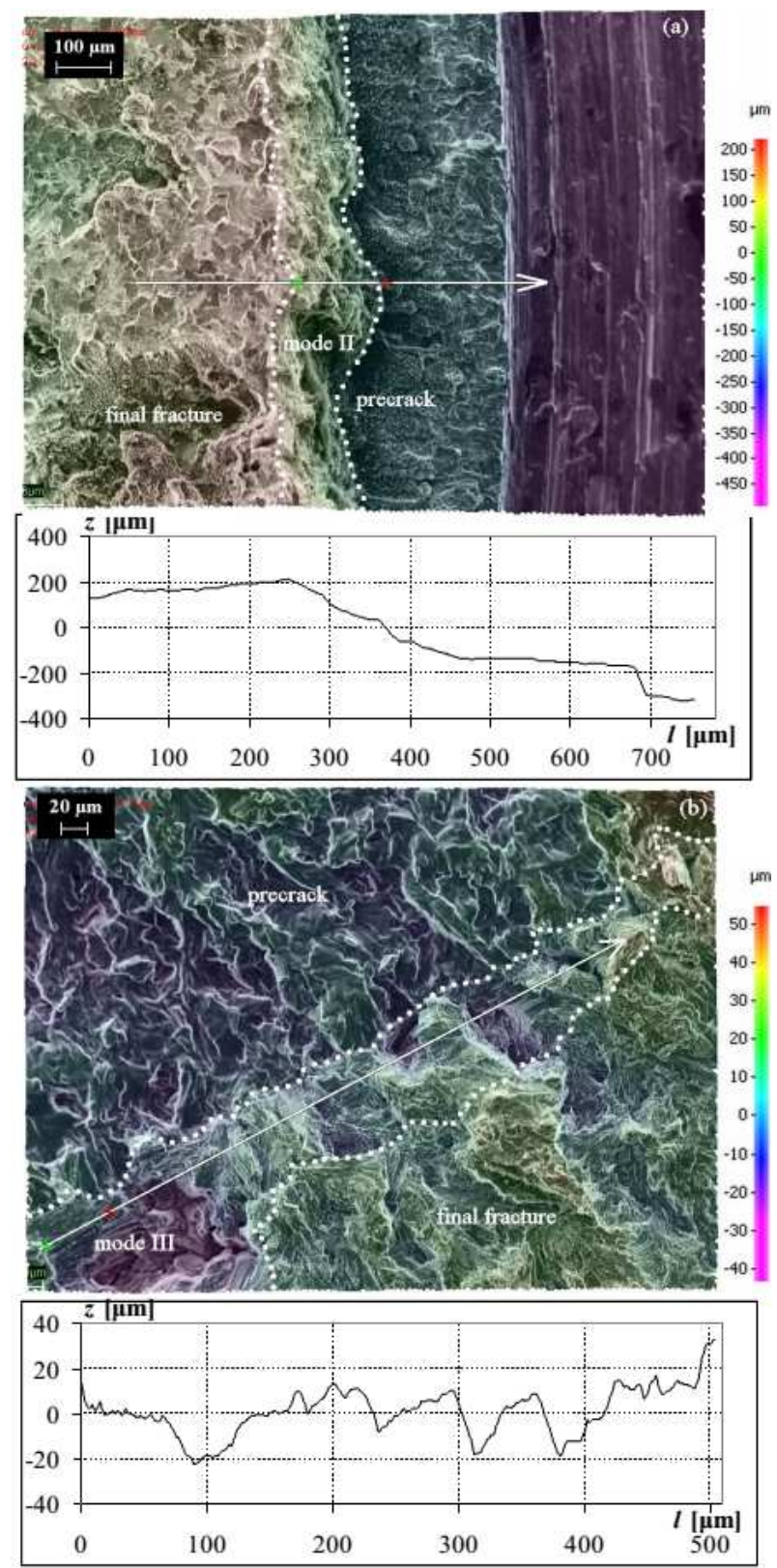

Fig. 3. Fracture surface morphology of a mode II crack (a) and a mode III crack (b) with the related height profiles and the third dimension $z$ according to the color codes. The white arrow defines the profile as well as the coordinate $l$ and it is parallel to the applied shear direction.

\section{Discussion}

In the remote mode II the cracks deflected by the mean angle $\alpha_{\mathrm{IIm}} \approx 50^{\circ}$ (see also Fig. 3) which corresponds to the ratio of the local SIFRs $\Delta K_{\mathrm{I}} / \Delta K_{\mathrm{II}} \approx 2$, i.e., to a propagation under a dominant opening mode [7]. This is significantly different from the mean angle $\alpha_{\mathrm{IIm}, \mathrm{A}} \approx 18^{\circ}$ ( $\Delta K_{\mathrm{I}} / \Delta K_{\mathrm{II}} \approx 0.5$, dominant shear mode) previously observed in the ARMCO iron. In the remote mode III, similarly, some highly twisted facets indicated a formation of the factory-roof-like morphology. This can be explained by the fact that the cementite lamellas in the pearlitic grains constitute a strong barriers to dislocation movement in slip systems and thus to the coplanar shear-mode crack growth. Instead, these dislocations are forced to bypass the barriers by the out-of-plane Orowan mechanism which promotes the local mode I branching.

The local stress intensity factor $K_{\text {I }}$ for a mode I branch at the crack front loaded in mode II can be calculated as $K_{\mathrm{I}}=1.15 K_{\mathrm{II}}$ [11]. Based on this relation one can check a simple criterion, Eq. (3), for growth of this mode I branch when knowing the effective thresholds $\Delta K_{\text {IIeff }}$ and $\Delta K_{\text {Ieff,th }}[7]$ :

$$
\Delta K_{\mathrm{I}}=1.15 \Delta K_{\mathrm{IIeff}, \mathrm{th}} \geq \Delta K_{\mathrm{Ieff}, \mathrm{th}} .
$$

For the studied ferritic-pearlitic steel the mode I effective threshold is $\Delta K_{\text {Ieff }, \text { th }}=3.0 \mathrm{MPa} \mathrm{m}^{1 / 2}$ [12]. Using this value and the measured value $\Delta K_{\text {IIeff,th }}=2.9 \mathrm{MPam}^{1 / 2}$ in Eq. (3) one obtains $3.3>3.0$, which shows that the deflection criterion is fulfilled. This is in agreement with fractographical observations revealing the crack growth under the dominance of mode I loading.

The mean angle $\alpha_{\mathrm{IIm}} \approx 50^{\circ}$ can also be used in the calculation of the effective threshold $\Delta K_{\text {IIeff,th ac- }}$ cording to Eq. (1). This gives the theoretical value of $3.1 \mathrm{MPam}^{1 / 2}$ which is close to the measured value of $2.9 \mathrm{MPa} \mathrm{m}^{1 / 2}$.

\section{Conclusions}

Experiments on fatigue crack propagation in modes II and III were done on ferritic-pearlitic steel. Analysis of fracture morphologies in three dimensions revealed that the remote mode II cracks deflected by the mean angle $\alpha_{\mathrm{IIm}} \approx 50^{\circ}$ to reach a dominant mode I loading, which is significantly different from $\alpha_{\mathrm{IIm}, \mathrm{A}} \approx 18^{\circ}$ (mode II dominance) as previously observed in the ARMCO iron. This is also in agreement with the result of a simple LEFMbased criterion for mode I branching. Similarly, the remote mode III fracture morphology indicated a formation of the factory-roof-like morphology. Effective thresholds were measured as follows: $\Delta K_{\text {IIeff,th }}=2.9 \mathrm{MPam}^{1 / 2}$ and $\Delta K_{\text {IIIeff,th }}=4.4 \mathrm{MPa} \mathrm{m}^{1 / 2}$. The equation for calculation of the mode II effective threshold gave the value of $3.1 \mathrm{MPam}^{1 / 2}$, which is close to the value found out experimentally.

\section{Acknowledgments}

The authors acknowledge the financial support of this work by the Czech Science Foundation in the frame of the project No. P108/12/0144 and by the European Regional Development Fund (CEITEC CZ.1.05/1.1.00/02.0068).

\section{References}

[1] R.O. Ritchie, Mater. Sci. Eng. A 15, 103 (1988).

[2] S. Suresh, Fatigue of Materials, Cambridge University Press, Cambridge, UK 1998. 
[3] E.K. Tschegg, R.O. Ritchie, F.A. McClintock, Int. J. Fatigue 5, 29 (1983).

[4] E.K. Tschegg, Acta Metall. 31, 1323 (1984).

[5] V. Doquet, S. Pommier, Fatigue Fract. Eng. Mater. Struct. 27, 1051 (2004).

[6] T. Vojtek, R. Pippan, A. Hohenwarter, L. Holán̆ J. Pokluda, Acta Mater. 61, 4625 (2013).

[7] J. Pokluda, R. Pippan, T. Vojtek, A. Hohenwarter, Fatigue Fract. Eng. Mater. Struct. 37, 232 (2014).

[8] J. Pokluda, R. Pippan, Fatigue Fract. Eng. Mater. Struct. 28, 179 (2005).
[9] H. Nayeb-Hashemi, F.A. McClintock, R.O. Ritchie, Int. J. Fracture 23, 163 (1983).

[10] M. Klesnil, P. Lukáš, Fatigue of Metallic Materials, Elsevier, Amsterdam 1992.

[11] L.P. Pook, Crack Paths, Wit Press, Southampton 2002.

[12] T. Billaudeau, Y. Nadot, G. Bezine, Acta Mater. 52, 3911 (2004). 